\title{
Safety of immune checkpoint inhibitors in patients with cancer and pre-existing autoimmune disease
}

\author{
Swetha Alexander ${ }^{1}$, Umang Swami ${ }^{2}$, Aneet Kaur ${ }^{3}$, Yubo Gao ${ }^{4}$, Munazza Fatima ${ }^{5}$, Meredith M. Ginn ${ }^{6}$, \\ Jill E. Stein ${ }^{6}$, Petros Grivas ${ }^{7}$, Yousef Zakharia ${ }^{6}$, Namrata Singh ${ }^{8}$
}

${ }^{1}$ Internal Medicine, University of Connecticut, CT, USA; ${ }^{2}$ Department of Internal Medicine, Division of Oncology, Huntsman Cancer Institute, University of Utah, Salt Lake City, UT, USA; ${ }^{3}$ Department of Rheumatology, MultiCare Rheumatology Specialists, Tacoma, WA, USA; ${ }^{4}$ Department of Internal Medicine, University of Iowa Hospitals and Clinics, Iowa City, IA, USA; ${ }^{5}$ Division of Rheumatology, University of Iowa Hospitals and Clinics, Iowa City, IA, USA; ${ }^{6}$ Department of Pharmaceutical Care, The University of Iowa Hospitals and Clinics, Iowa City, IA, USA; ${ }^{7}$ Division of Medical Oncology, Department of Medicine, University of Washington, Seattle Cancer Care Alliance, Fred Hutchinson Cancer Research Center, Seattle, WA, USA; ${ }^{8}$ Division of Rheumatology, University of Washington, Seattle, WA, USA

Contributions: (I) Conception and design: U Swami, A Kaur, M Fatima, N Singh; (II) Administrative support: N Singh; (III) Provision of study materials or patients: U Swami, A Kaur, M Fatima, MM Ginn, JE Stein, Y Zakharia; (IV) Collection and assembly of data: U Swami, A Kaur, M Fatima, N Singh; (V) Data analysis and interpretation: All authors; (VI) Manuscript writing: All authors; (VII) Final approval of manuscript: All authors.

Correspondence to: Namrata Singh, MD MSCI. 1959 NE Pacific Street, Room\#BB561, Seattle, WA 98195, USA. Email: nasingh@uw.edu.

Background: Patients with pre-existing autoimmune disease (AD) have been largely excluded from clinical trials of immune checkpoint inhibitors (ICI), so data on safety of ICIs among patients with pre-existing AD are relatively limited. There is a need for deeper understanding of the type and management of complications from ICI in patients with pre-existing AD. We sought to investigate the safety of ICIs in patients with pre-existing $\mathrm{ADs}$ as well as factors associated with $\mathrm{AD}$ flare.

Methods: Consecutive patients with pre-existing AD who received monotherapy as well as combination of ICI therapies at our institution from September 2015 through September $1^{\text {st }}$, 2018 were identified. Clinical information was abstracted via manual chart review. Clinical factors associated with AD flare were determined using multivariable logistic regression.

Results: A total of 42 patients were identified of whom 12 developed AD flare. All flares were treated with oral or topical corticosteroids, while a patient with flare of rheumatoid arthritis was treated with tofacitinib and another patient with Crohn's flare was treated with infliximab. Female sex, smoking status, higher age at the start of ICI therapy, cancer type, such as melanoma and lung cancer as compared to other cancers, were not significantly associated with $\mathrm{AD}$ flare, however, patients with underlying rheumatologic $\mathrm{AD}$ were noted to have a five times greater likelihood of flare as compared to other non-rheumatologic AD. Nine patients developed new immune related adverse events (IRAEs) unrelated to underlying AD, such as inflammatory poly-arthropathy, neuropathy, hypothyroidism, diarrhea, lichenoid drug eruptions, which were managed with oral and/or topical corticosteroids. ICI was stopped in six patients due to AD flare, in four patients due to IRAE flare (out of which one resumed ICI after resolution of IRAE).

Conclusions: In patients with pre-existing AD treated with ICI, AD flare occurred in $28 \%$ of patients and were managed successfully with corticosteroids alone or with additional disease-modifying therapies. ICI could be considered in patients with $\mathrm{AD}$, but with very close monitoring and preemptive multidisciplinary collaboration.

Keywords: Immune checkpoint inhibitors (ICI); immune related adverse events (IRAEs); autoimmune disease (AD); immunotherapy; immune toxicity

Submitted Dec 24, 2020. Accepted for publication Mar 25, 2021.

doi: 10.21037/atm-20-8124

View this article at: http://dx.doi.org/10.21037/atm-20-8124 


\section{Introduction}

Immune checkpoint inhibitors (ICI), such as monoclonal antibodies directed against cytotoxic T-lymphocyteassociated protein 4 (CTLA-4), programmed cell death protein 1 (PD-1), and programmed cell death ligand 1 (PDL1) have been approved for use in advanced and metastatic cancers, ushering new therapeutic options in the field of immuno-oncology. The side effects of these agents appear to manifest due to the unchecked activity of $\mathrm{T}$ cells and causes them to damage the organ systems due to immuneactivated inflammation, called immune-related adverse events (IRAEs) (1). The commonly affected organ systems include skin, gastrointestinal tract, endocrine, liver, lung, kidney, musculoskeletal, among many others (2).

During the early clinical trials of ICI, patients with autoimmune disease (AD) have largely been excluded, due to the perceived risk of frequent IRAEs, which can range from mild to life-threatening and/or possible flare of the underlying $\mathrm{AD}$ (3-13). Due to this, there is a knowledge gap regarding the safety of ICI therapy among patients with pre-existing $\mathrm{AD}$ who develop cancer.

The role of ICI continues to expand in different types of cancer with emerging data. This has led to a growing need for more robust safety data among patients with $\mathrm{AD}$ and cancer who need to be treated with ICI. Therefore, we conducted this single-center, observational retrospective study to evaluate the occurrence of $\mathrm{AD}$ flares and/or new IRAEs among patients with prior ADs treated with ICI and identify the factors associated with increased AD flare such as rheumatological $\mathrm{AD}$ as compared to non-rheumatological AD. We present the following article in accordance with the STROBE reporting checklist (available at http://dx.doi. org/10.21037/atm-20-8124).

\section{Methods}

We retrospectively collected clinical and pharmacological data on all patients with cancer who received ICI and had a pre-existing $\mathrm{AD}$, at the University of Iowa Hospitals and Clinics from September 2015 through September 2018. Patients with pre-existing AD were identified by independent chart review by three co-authors (AK, US, $M F)$. The ICI inhibitors included in the study were antiPD-1 agents (Pembrolizumab and Nivolumab); anti-PD-L1 agent (Atezolizumab) and CTLA-4 inhibitor (Ipilimumab). Variables recorded during systematic comprehensive chart review were: nature (type) of the preexisting $\mathrm{AD}$ (rheumatologic versus other), activity (activity was defined based on the patient's symptoms and treating physician's assessment) of the $\mathrm{AD}$ (in remission versus active); previous and current therapies for $\mathrm{AD}$; timeline of diagnosis of $\mathrm{AD}$ and cancer; type of ICI therapy given (which included monotherapy as well combination), course and duration of ICI therapy; timeline of development of flares of preexisting $\mathrm{AD}$ (if applicable); development of any IRAE and if so, grade/severity and course of the patient's IRAE; and vital status at the time of last documentation available in the chart. Progression free survival (PFS) was defined as the time from start of ICI to progression as per RECIST 1.1 , clinical progression or death. Overall survival (OS) was defined as the time from start of ICI to death from any cause. Among patients who did not progress or die during follow-up, censoring time was defined as the time of most recent follow up. The study was conducted in accordance with the Declaration of Helsinki (as revised in 2013). The study was approved by institutional review board of the University of Iowa (IRB\# 201808794) and individual consent for this retrospective analysis was waived.

Descriptive statistics were used to describe the baseline characteristics of the cohort. Multivariable logistic regression was used to identify the predictors of developing $\mathrm{AD}$ flare. Clinical factors of interest selected were: age, sex, smoking status, cancer type (metastatic melanoma, lung cancer $v s$. others) and rheumatologic $\mathrm{AD} v s$. others. We considered a p-value less than 0.05 to be significant and the analysis was conducted using statistical analysis software (SAS) V.9.4.

\section{Results}

\section{Study population}

A total of 917 patients with cancer were identified who received an ICI therapy during the study time period. Of these, 42 patients were eligible for inclusion due to having a pre-existing $\mathrm{AD}$ and treatment with an ICI for underlying cancer. The types of cancer for which ICI therapy was given are listed in Table 1. The underlying ADs identified are listed in Table 2. The baseline characteristics of the participants are included in Table 1. Most participants were men $[24 / 42(57 \%)]$ with the most frequent cancer treated with an ICI being metastatic melanoma [25/42 (60\%)]. There was a high proportion of patients who smoked [25/42 $(60 \%)]$, and pembrolizumab [23/42 (55\%)] and nivolumab [13/42 (31\%)] were the more commonly used ICIs as compared to other ICI drugs. The most frequent $\mathrm{AD}$ in our 
Table 1 Baseline characteristics of the cohort

\begin{tabular}{lc}
\hline Characteristic & Total $(\mathrm{n}=42)$ \\
\hline Gender & 24 \\
Male & 18 \\
Female & \\
Type of cancer & 25 \\
Metastatic melanoma & 11 \\
Lung cancer & 4 \\
Genitourinary (urothelial and renal) & 1 \\
Diffuse large B-cell lymphoma & 1 \\
Head and neck & $67[13]$ \\
Mean [SD] age at diagnosis of cancer & \\
Smoking status & 25 \\
Ever smoker & 17 \\
Never smoker & 13 \\
Immune checkpoint inhibitors used & 1 \\
Pembrolizumab & 23 \\
Nivolumab & \\
Atezolizumab & \\
\hline Ipilimumab & \\
\hline
\end{tabular}

cohort was psoriasis $(6 / 42)$ followed by rheumatoid arthritis $(5 / 42)$ and polymyalgia rheumatica (5/42).

Table 2 shows the AD identified among those who received ICI therapy, $40 \%$ of patients had a pre-existing rheumatologic condition, $24 \%$ had dermatologic, $12 \%$ had neurologic, $9.5 \%$ had endocrine and, $9.5 \%$ had other miscellaneous conditions (immune thrombocytopenic purpura, membranoproliferative glomerulonephritis and pulmonary sarcoidosis), and $5 \%$ had inflammatory bowel disease. Table 3 demonstrates baseline treatment for preexisting $\mathrm{AD}$ before the initiation of ICI therapy; 14 out of 42 were not on any treatment for their pre-existing $\mathrm{AD}$ at the time of start of ICI therapy. All patients had stable AD at the time of ICI therapy initiation.

\section{AD flare during ICI therapy}

Overall, 12 (28\%) of patients developed AD flare during ICI therapy (Table 4). The median time to $\mathrm{AD}$ flare among those who flared was 68 days. The average age at the time of ICI initiation among those who flared was 70.7 (11.8) years and
Table 2 Autoimmune disease frequency identified who received ICI therapy

\begin{tabular}{|c|c|}
\hline Autoimmune disease & Frequency $(n=42)$ \\
\hline Rheumatologic & $17(40 \%)$ \\
\hline 1. Rheumatoid arthritis & 5 \\
\hline 2. Polymyalgia rheumatica & 5 \\
\hline 3. Psoriatic arthritis & 4 \\
\hline 4. Inflammatory arthritis & 1 \\
\hline 5. Scleroderma & 1 \\
\hline 6. Henoch Schönlein purpura & 1 \\
\hline Dermatologic & $10(24 \%)$ \\
\hline 1. Psoriasis & 6 \\
\hline 2. Cutaneous lupus & 2 \\
\hline 3. Vitiligo & 1 \\
\hline 4. Bullous pemphigoid & 1 \\
\hline Endocrine & $4(10 \%)$ \\
\hline 1. Hashimoto's thyroiditis & 1 \\
\hline 2. Graves disease & 1 \\
\hline 3. Autoimmune hypophysitis & 1 \\
\hline 4. Type 1 diabetes mellitus & 1 \\
\hline Gastrointestinal & $2(5 \%)$ \\
\hline 1. Crohn's & 1 \\
\hline 2. Ulcerative colitis & 1 \\
\hline Neurologic & $5(12 \%)$ \\
\hline 1. Guillain Barre & 2 \\
\hline 2. Myasthenia gravis & 1 \\
\hline 3. Multiple sclerosis & 1 \\
\hline $\begin{array}{l}\text { 4. Chronic inflammatory demyelinating } \\
\text { neuropathy }\end{array}$ & 1 \\
\hline Others & $4(10 \%)$ \\
\hline 1. Immune thrombocytopenic purpura (ITP) & 2 \\
\hline 2. Pulmonary sarcoidosis & 1 \\
\hline $\begin{array}{l}\text { 3. Membranoproliferative glomerulonephritis } \\
\text { (MPGN) }\end{array}$ & 1 \\
\hline
\end{tabular}

among those who did not flare was 67.1 (13). Seven patients developed grade 1-2 flare, three developed grade 3-4, while two flares were unable to be graded. Eight were treated only with corticosteroid therapy out of which six received oral 
Table 3 Baseline treatment for pre-existing autoimmune disease (AD) before initiation of Immune check point inhibitor therapy

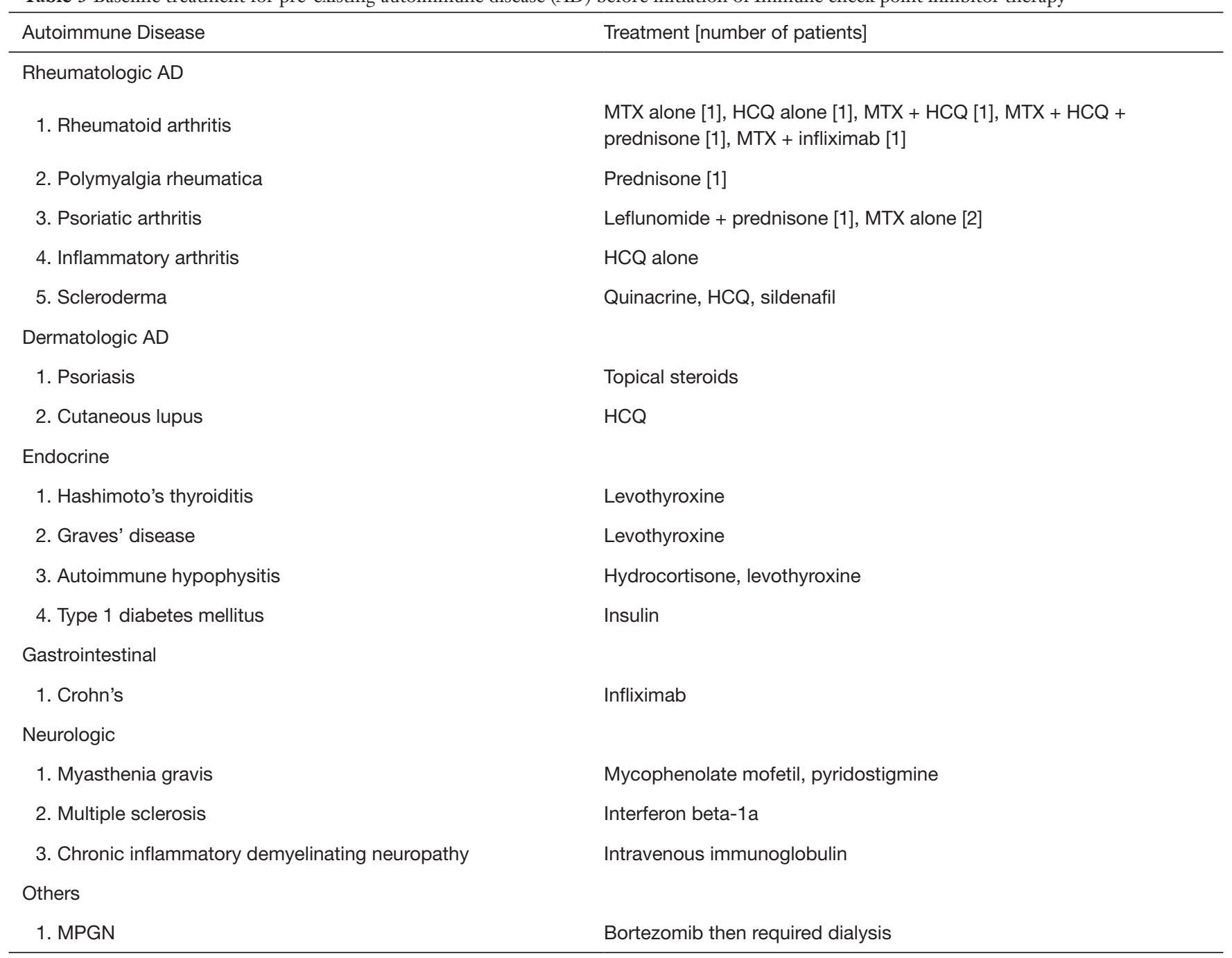

$\mathrm{AD}$, autoimmune disease; HCQ, hydroxychloroquine; MTX, methotrexate; MPGN, membranoproliferative glomerulonephritis.

glucocorticoids, two required topical steroids and one required intravenous high dose steroid. One patient recovered only with best supportive therapy. One patient required tofacitinib and another infliximab to treat $\mathrm{AD}$ exacerbation. One patient required plasmapheresis, pyridostigmine, rituximab, mycophenolic acid, abatacept and oral prednisone for a grade 4 $\mathrm{AD}$ flare. Due to the $\mathrm{AD}$ flare, five patients had to permanently discontinue the ICI therapy. One patient who also developed an $\mathrm{AD}$ had to stop therapy due to non-response to therapy and not related to the $\mathrm{AD}$ flare.

\section{Factors associated with AD Flare}

Results of the multivariable logistic regression to identify factors associated with $\mathrm{AD}$ flare are shown in Table 5. Smoking status (OR 1.793; 95\% CI: 0.353-9.118), age at the start of ICI therapy (OR 1.013; 95\% CI: 0.950-1.08), being obese (OR 1.684; 95\% CI: 0.207-13.724) and particular cancer types, such as melanoma and lung cancer compared to others (OR 1.277; 95\% CI: 0.23-6.96), female sex (OR 0.81 ; $95 \%$ CI: $0.165-3.97)$ were not significantly associated with $\mathrm{AD}$ flare. Patients with an underlying rheumatologic $\mathrm{AD}$ were noted to have a significantly higher occurrence (OR 5.678; 95\% CI: 1.10-29.299) of flare than patients with non-rheumatologic AD. Median PFS for melanoma was 31 months and for lung cancer was 5.3 months. Median OS for melanoma was not reached and for lung cancer was 12 months. 
Table 4 AD exacerbation during ICI therapy

\begin{tabular}{ll}
\hline Characteristic & Patients (n) \\
\hline Flare of underlying AD & 30 \\
1. Patients who did not develop AD flare & 12 \\
2. Patients who developed AD flare & \\
Grades of AD flares & 7 \\
1. Grade 1-2 & 3 \\
2. Grade 3-4 & 2 \\
3. Grade unknown & 1 \\
Treatment required for AD flares & 8 \\
1. No treatment required/supportive care & 1 \\
2. Corticosteroids only & 1 \\
3. Tofacitinib & 1 \\
4. Infliximab & \\
5. Plasmapheresis & \\
ICl therapy during/after AD flare & \\
- Continued & \\
- Permanently discontinued & \\
\hline
\end{tabular}

$A D$, autoimmune disease; ICI, Immune check point inhibitor.

Table 5 Factors associated with an AD flare while on ICI

\begin{tabular}{|c|c|c|}
\hline Variable & Odds ratio & $95 \% \mathrm{Cl}$ \\
\hline Sex (female vs. male) & 0.811 & $0.170-3.978$ \\
\hline $\begin{array}{l}\text { Smoking status (ever vs. never } \\
\text { smoker) }\end{array}$ & 1.793 & $0.353-9.118$ \\
\hline Age at the start of $\mathrm{ICI}$ therapy & 1.013 & $0.950-1.080$ \\
\hline Obese (versus not) & 1.684 & $0.207-13.724$ \\
\hline $\begin{array}{l}\text { Underlying rheumatologic condition } \\
\text { (yes vs. no) }\end{array}$ & 5.678 & 1.100-29.299 \\
\hline $\begin{array}{l}\text { Type of cancer (metastatic melanoma } \\
\text { and lung cancer vs. others) }\end{array}$ & 1.277 & $0.234-6.958$ \\
\hline
\end{tabular}

$A D$, autoimmune disease; $I C I$, Immune check point inhibitor.

\section{Immune related adverse events}

About 21\% (9/42) developed a new IRAE while on ICI as depicted in Table 6. IRAEs were noted in three patients with skin rash, two with inflammatory arthritis and one patient each with inflammatory arthritis, colitis, neuropathy and hypothyroidism. Among them, 56\% developed grade 1-
Table 6 Development of immune related adverse events

\begin{tabular}{ll}
\hline Characteristic & Patients (n) \\
\hline IRAE unrelated to underlying AD & 33 \\
- Patients who did not develop IRAE's & 9 \\
- Patients who developed IRAE's & \\
IRAE's experienced among 9 patients & 5 \\
- Grade 1-2 & 4 \\
- Grade 3-4 & \\
ICI therapy during/after IRAE & 4 \\
- Continued & 1 \\
- Temporarily discontinued & 4 \\
- Permanently discontinued &
\end{tabular}

IRAE(s), immune related adverse event(s); $A D$, autoimmune disease; $\mathrm{ICl}$, immune checkpoint inhibitor; $n$, number of patients.

2 events and the rest developed grade 3-4 events. Four of the nine patients continued with ICI therapy despite IRAE, one patient required a temporary discontinuation and then resumed, while the rest (4/9) required a permanent discontinuation of ICI therapy.

\section{Discussion}

In our study, we found that among patients with a preexisting $\mathrm{AD}$ who received ICI therapy for cancer, a $28 \%$ developed AD flare and $21 \%$ developed a de novo IRAE with most being able to continue ICI therapy after treatment of the flare or IRAE. There remains an unmet need among AD population for treatment of cancer as the risk of developing malignancy among patients with $\mathrm{AD}$, especially rheumatoid arthritis, is higher than the general population.

The most frequent $\mathrm{AD}$ in our cohort was psoriasis $(6 / 42)$ followed by rheumatoid arthritis (5/42) and polymyalgia rheumatica (5/42), which appears to be consistent with a prior systematic review $(\mathrm{n}=123)$ (14). Among the studied population, about one-third (29\%) developed a flare of the pre-existing $\mathrm{AD}$ while on ICI therapy, this appears to be similar to other studies (15-19). Most AD flares were deemed grade $1-2$ (58\%), and were able to be managed with corticosteroids. Leonardi et al. reported $87 \%$, while Menzies et al. reported that $94 \%$ of patients in their studies developed grade 1-2 AD flare, suggestive of the possibility that among the patients who develop an $\mathrm{AD}$ flare, most of them are low grade and easily managed with corticosteroid 
therapy. More than half of the patients in our study went on to finish the ICI therapy despite the flare. Patients with rheumatologic $\mathrm{AD}$ were noted to have five times increased occurrence of $\mathrm{AD}$ flare as compared to patients with nonrheumatologic AD. This was also noted in Leonardi et al. study, however our study included more diverse types of malignancies and ICI therapies. Notably, other studies have also shown IRAEs to occur in less than a third of the studied population with most being able to complete ICI therapy despite IRAE development $(14,18,20,21)$.

Strengths of our study include the analysis of patients with various $\mathrm{AD}$ at baseline managed in a large academic center, as well as the analysis of several ICI, and a detailed systematic chart review. The limitations of our study include a retrospective single-center cohort study with small sample size, number of events, as well as missing data, e.g., a number of $\mathrm{AD}$ features may not have been captured with detailed granularity noted in clinical trials. The statistical analyses for the evaluation of factors associated with $\mathrm{AD}$ flare were underpowered given the small study sample. All our patients had stable $\mathrm{AD}$ at baseline, hence the results and interpretations may not be generalizable to patients with severe $\mathrm{AD}$ who need to be started on an ICI therapy. There was variability in the anti-cancer therapy course/ duration, monitoring schedule and follow up, as well as management of $\mathrm{AD}$ flares and IRAEs, which may impact result interpretation. This noted variability may introduce selection bias and confounding, which may be further addressed with larger sample size, longer follow up and landmark analyses, also adjusting for treatment duration, in future studies.

Our study helps evaluate the safety of ICI therapies in patients with cancer and pre-existing $\mathrm{AD}$, and showed only a minority developed AD flare or IRAE, which can usually be managed successfully (14-20,22-24). There has been evidence to suggest that a selective immunosuppressant agent be used for patients with active $\mathrm{AD}$ before treatment with ICI to prevent an $\mathrm{AD}$ exacerbation or even after development of IRAE in a multifaceted setting adept to manage this $(25,26)$. A multidisciplinary approach is strongly recommended when treating and monitoring these patents, while larger analyses can provide more definitive data and support the development of guidelines (27).

\section{Acknowledgements}

Funding: None.

\section{Footnote}

Provenance and Peer Review: This article was commissioned by the editorial office, Annals of Translational Medicine for the series "Cancer Immunotherapy: Recent Advances and Challenges". The article has undergone external peer review.

Reporting Checklist: The authors have completed the STROBE reporting checklist. Available at http://dx.doi. org/10.21037/atm-20-8124

Data Sharing Statement: Available at http://dx.doi. org/10.21037/atm-20-8124

Conflicts of Interest: All authors have completed the ICMJE uniform disclosure form (available at http:// dx.doi.org/10.21037/atm-20-8124). The series "Cancer Immunotherapy: Recent Advances and Challenges" was commissioned by the editorial office without any funding or sponsorship. Umang Swami served as the unpaid Guest Editor of the series and serves as an unpaid editorial board member of Annals of Translational Medicine from May 2019 to Apr 2021. Dr. PG reports funding to institutions from Merck, Mirati Therapeutics, Pfizer, Clovis Oncology, Bavarian Nordic, Immunomedics, Debiopharm, Bristol-Myers Squibb, QED Therapeutics, GlaxoSmithKline, and Kure IT Cancer Research; consulting fees from AstraZeneca, Bayer, Bristol-Myers Squibb, Clovis Oncology, Dyania Health, Driver, EMD Serono, Exelixis, Foundation Medicine, Genentech/ Roche, Genzyme, GlaxoSmithKline, Heron Therapeutics, Immunomedics, Infinity Pharmaceuticals, Janssen, Merck, Mirati Therapeutics, Pfizer, Seattle Genetics, and QED Therapeutics; and travel support from AstraZeneca and Clovis Oncology. Dr. YZ reports institution clinical trial support from NewLink Genetics, Pfizer, Exelixis, and Eisai; consultant honorarium from Pfizer and Novartis; and that he is an Advisory Board member of Amgen, Roche Diagnostics, Novartis, Janssen, Eisai, Exelixis, Castle Bioscience, Array, Bayer, Pfizer, Clovis, and EMD serono, and a Data and Safety Monitoring Committee member of Janssen Research and Development. NS is supported by grants from the Rheumatology Research Foundation and the American Heart Association. The authors have no other conflicts of interest to declare.

Ethical Statement: The authors are accountable for all 
aspects of the work in ensuring that questions related to the accuracy or integrity of any part of the work are appropriately investigated and resolved. The study was conducted in accordance with the Declaration of Helsinki (as revised in 2013). The study was approved by institutional review board of the University of Iowa (IRB\# 201808794) and individual consent for this retrospective analysis was waived.

Open Access Statement: This is an Open Access article distributed in accordance with the Creative Commons Attribution-NonCommercial-NoDerivs 4.0 International License (CC BY-NC-ND 4.0), which permits the noncommercial replication and distribution of the article with the strict proviso that no changes or edits are made and the original work is properly cited (including links to both the formal publication through the relevant DOI and the license). See: https://creativecommons.org/licenses/by-nc-nd/4.0/.

\section{References}

1. Postow MA, Sidlow R, Hellmann MD. Immune-related adverse events associated with immune checkpoint blockade. N Engl J Med 2018;378:158-68.

2. Wang P-F, Chen Y, Song S-Y, et al. Immune-related adverse events associated with anti-PD-1/PD-L1 treatment for malignancies: a meta-analysis. Front Pharmacol 2017;8:730.

3. Abdel-Wahab N, Shah M, Suarez-Almazor ME. Adverse events associated with immune checkpoint blockade in patients with cancer: a systematic review of case reports. PloS One 2016;11:e0160221.

4. Bertrand A, Kostine M, Barnetche T, et al. Immune related adverse events associated with anti-CTLA-4 antibodies: systematic review and meta-analysis. BMC Med 2015;13:211.

5. Borghaei H, Paz-Ares L, Horn L, et al. Nivolumab versus docetaxel in advanced nonsquamous non-small-cell lung cancer. N Engl J Med 2015;373:1627-39.

6. Boutros C, Tarhini A, Routier E, et al. Safety profiles of anti-CTLA-4 and anti-PD-1 antibodies alone and in combination. Nat Rev Clin Oncol 2016;13:473-86.

7. Garon EB, Rizvi NA, Hui R, et al. Pembrolizumab for the treatment of non-small-cell lung cancer. N Engl J Med 2015;372:2018-28.

8. Hodi FS, O'Day SJ, McDermott DF, et al. Improved survival with ipilimumab in patients with metastatic melanoma. N Engl J Med 2010;363:711-23.
9. Johnson DB, Friedman DL, Berry E, et al. Survivorship in immune therapy: assessing chronic immune toxicities, health outcomes, and functional status among long-term ipilimumab survivors at a single referral center. Cancer Immunol Res 2015;3:464-9.

10. Larkin J, Chiarion-Sileni V, Gonzalez R, et al. Combined nivolumab and ipilimumab or monotherapy in untreated melanoma. N Engl J Med 2015;373:23-34.

11. Michot JM, Bigenwald C, Champiat S, et al. Immunerelated adverse events with immune checkpoint blockade: a comprehensive review. Eur J Cancer 2016;54:139-48.

12. Rosenberg JE, Hoffman-Censits J, Powles T, et al. Atezolizumab in patients with locally advanced and metastatic urothelial carcinoma who have progressed following treatment with platinum-based chemotherapy: a single-arm, multicentre, phase 2 trial. Lancet 2016;387:1909-20.

13. Tocut M, Brenner R, Zandman-Goddard G. Autoimmune phenomena and disease in cancer patients treated with immune checkpoint inhibitors. Autoimmun Rev 2018;17:610-6.

14. Abdel-Wahab N, Shah M, Lopez-Olivo MA, et al. Use of immune checkpoint inhibitors in the treatment of patients with cancer and preexisting autoimmune disease: a systematic review. Ann Intern Med 2018;168:121-30.

15. Danlos FX, Voisin AL, Dyevre V, et al. Safety and efficacy of anti-programmed death 1 antibodies in patients with cancer and pre-existing autoimmune or inflammatory disease. Eur J Cancer 2018;91:21-9.

16. Johnson DB, Sullivan RJ, Ott PA, et al. Ipilimumab therapy in patients with advanced melanoma and preexisting autoimmune disorders. JAMA Oncol 2016;2:234-40.

17. Kähler KC, Eigentler TK, Gesierich A, et al. Ipilimumab in metastatic melanoma patients with pre-existing autoimmune disorders. Cancer Immunol Immunother 2018;67:825-34.

18. Leonardi GC, Gainor JF, Altan M, et al. Safety of programmed Death-1 pathway inhibitors among patients with Non-Small-Cell lung cancer and preexisting autoimmune disorders. J Clin Oncol 2018;36:1905.

19. Menzies AM, Johnson DB, Ramanujam S, et al. AntiPD-1 therapy in patients with advanced melanoma and preexisting autoimmune disorders $(\mathrm{AD})$ or major toxicity with ipilimumab (IPI). American Society of Clinical Oncology, 2016.

20. Gutzmer R, Koop A, Meier F, et al. Programmed cell death protein-1 (PD-1) inhibitor therapy in patients with advanced melanoma and preexisting autoimmunity 
or ipilimumab-triggered autoimmunity. Eur J Cancer 2017;75:24-32.

21. Kennedy LC, Bhatia S, Thompson JA, et al. Preexisting autoimmune disease: implications for immune checkpoint inhibitor therapy in solid tumors. J Natl Compr Canc Netw 2019;17:750-7.

22. Lee B, Wong A, Kee D, et al. The use of ipilimumab in patients with rheumatoid arthritis and metastatic melanoma. Ann Oncol 2016;27:1174-7.

23. Richter MD, Pinkston O, Kottschade LA, et al. Brief report: cancer immunotherapy in patients with preexisting rheumatic disease: the Mayo Clinic experience. Arthritis Rheumatol 2018;70:356-60.

24. Tison A, Quéré G, Misery L, et al. Safety and efficacy of immune checkpoint inhibitors in patients with cancer and

Cite this article as: Alexander S, Swami U, Kaur A, Gao Y, Fatima M, Ginn MM, Stein JE, Grivas P, Zakharia Y, Singh N. Safety of immune checkpoint inhibitors in patients with cancer and pre-existing autoimmune disease. Ann Transl Med 2021;9(12):1033. doi: 10.21037/atm-20-8124 preexisting autoimmune disease: a nationwide, multicenter cohort study. Arthritis Rheumatol 2019;71:2100-11.

25. Haanen J, Ernstoff MS, Wang Y, et al. Autoimmune diseases and immune-checkpoint inhibitors for cancer therapy: review of the literature and personalized riskbased prevention strategy. Ann Oncol 2020;31:724-44.

26. Haanen J, Ernstoff M, Wang Y, et al. Rechallenge patients with immune checkpoint inhibitors following severe immune-related adverse events: review of the literature and suggested prophylactic strategy. J Immunother Cancer 2020;8:e000604.

27. Kennedy LC, Wong KM, Kamat NV, et al. Untangling the Multidisciplinary Care Web: Streamlining Care Through an Immune-Related Adverse Events (IRAE) Tumor Board. Target Oncol 2020;15:541-8. 